\title{
Power Analysis of Hydraulic-pneumatic Booster on an Air Automobile based on Dimensionless Method
}

\author{
Xiangheng $\mathrm{Fu}^{1, \text { a }}$ \\ ${ }^{1}$ School of Automation Science and Electrical Engineering, Beihang University, Beijing, CHINA \\ a'E-mail: fxheng@163.com
}

Keywords: Power analysis. Dimensionless method. Hydraulic-pneumatic. Efficiency.

\begin{abstract}
In this study, to illustrate the energy conversion characteristics of air-powered hydraulic vehicle, dimensionless mathematical model of the working process is set up. Then the dynamic power and efficiency are researched based on the dimensionless parameter simulation. This research can be referred to in the performance and design optimization of the HP transformers.
\end{abstract}

\section{Introduction}

Hydropneumatic transformer (short for HP) is a machine which is used to convert the energy of compressed air into hydraulic oil's. Because of its small size, simple structure and applicability, it is widely used in all kinds of situation, such as in the aspects of petroleum chemical industry, new energy vehicle and medical apparatus and instruments [1-2]. In order to optimize performance, some study has been done. Shen et al study the aerodynamic performance of the intake air [3], and Professor Shi in Beihang University establishes the dimensionless model and equations of HP transformer in his paper [4]. Takeuchi at el design a new type of pump, which uses the expansion energy, and they study the relationship between the efficiency and some key parameters [5]. We have explored on hydropneumatic transformer for designing guidance and performance optimization.

In this paper, we introduce the structure of HP transformer firstly and then, the principle of it is introduced in detail. Based on the principle, the mathematical model is expressed correctly, including dimensionless energy equation, dimensionless continuity equation and so on. And according to the mathematic model, we establish the simulation program by software MATLAB/Simulation. To verify the correctness of the mathematical model, the physical object is made and the experiment platform is set up. The results of experiment prove the model is correct. Then we explore the key parameters that influence the efficiency and output power, including the dimensionless input pressure of compressed air, dimensionless stroke of the piston and dimensionless air area of piston. The results show that with the increase of the dimensionless input pressure and dimensionless air area, the output power increases too; but the output power decreases when dimensionless stroke increases. We can also make a conclusion that the dimensionless input pressure ranges from $\mathrm{A}$ to $\mathrm{B}$ and the values of dimensionless air area is between $\mathrm{A}$ and $\mathrm{B}$, the efficiency of HP transformer is reached at A\% or more. And the dimensionless stroke of the piston varies, the efficiency keeps the constant nearly, and this is because the stroke of the trip has little effect on energy loss.

\section{Structure and Working Principles of the HP Transformer}

As we can see from figure1, a typical hydraulic-pneumatic transformer is composed of air source, pressure regulator, reversing valve, check valve, pneumatic drive chambers, hydraulic pump chamber and so on.

When it starts working, the compressed air in the air source will flow into the pneumatic chamber A through the check valve, and the oil flows into the hydraulic chamber B at the same time. The pneumatic chamber B is connected to the atmosphere and the hydraulic chamber A is empty. The force on the piston from the pneumatic and hydraulic chambers is not equal, so the piston will 
move to the hydraulic chamber B until reaching its end-of-stroke. In this process, if the pressure of oil is equal to output pressure, the check valve will be opened and the high pressure oil flows out of the chamber.

The revering valve will change the state when the piston reaches its terminus and impacts the reversing valve. After that, the pneumatic chamber A will be connected to the atmosphere and the compressed air flows into the pneumatic chamber B, while the hydraulic chamber A is full of the oil and hydraulic chamber B is empty. The force on the piston is not balance again, so it will move to hydraulic chamber A. When the pressure of the oil in hydraulic chamber A is equal to out pressure, the oil will flow out though the check valve. And the state of revering valve will be changed again when the piston move to its end-of-stroke. And the oil will flow out of the hydraulic chambers through repeating the process mentioned above.

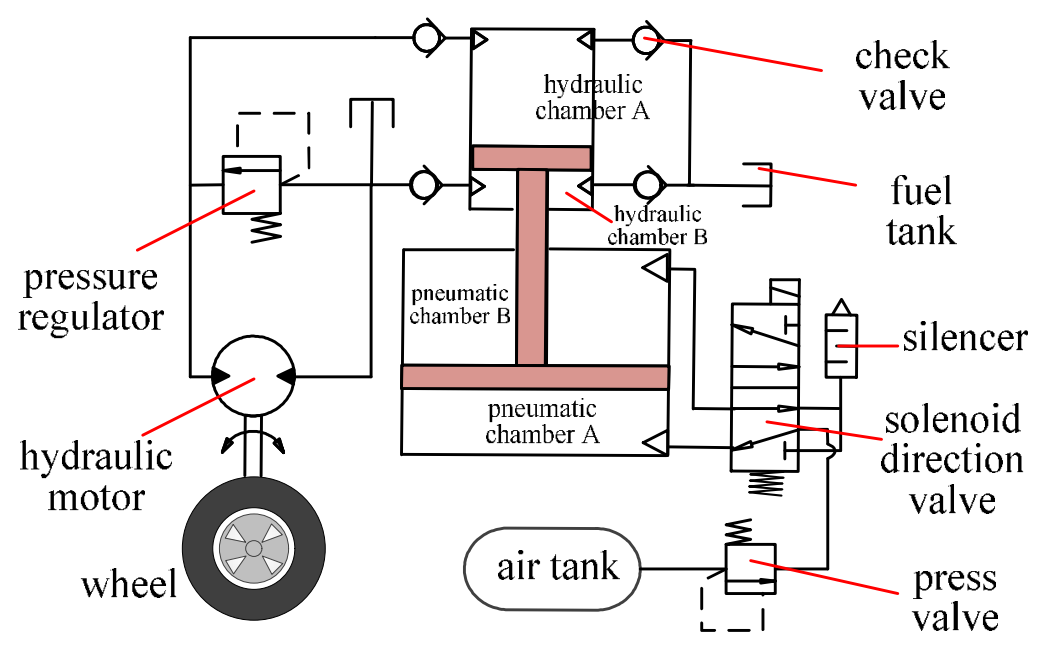

Fig.1. Structure of the power system of air-powered vehicle

\section{Mathematical formulation}

\section{Reference values and dimensionless variables}

Table 1 Reference values and dimensionless variables

\begin{tabular}{|c|c|c|c|}
\hline Variable & \multicolumn{2}{|c|}{ Reference value } & $\begin{array}{l}\text { Dimensionless } \\
\text { Variable }\end{array}$ \\
\hline Affective area & $A_{p}$ & $\begin{array}{c}\text { Aera of the piston of the pneumatic } \\
\text { chamber }\end{array}$ & $A_{e}^{*}=\frac{A_{e}}{A_{p}}$ \\
\hline Time & $T_{\min }$ & $\begin{array}{l}\text { Time to totally exhaust } W_{b} \text { of air at } G_{\max } \\
\text { of air mass flow }\end{array}$ & $T^{*}=\frac{t}{T_{\min }}$ \\
\hline Velocity & $\bar{u}=\frac{L}{T_{\min }}$ & Average velocity & $u^{*}=\frac{u}{-}$ \\
\hline Pressure & $P_{s}$ & Supply pressure & $P^{*}=\frac{P}{P_{s}}$ \\
\hline Temperature & $\theta_{a}$ & Atmosphere temperature & $\theta^{*}=\frac{\theta}{\theta_{a}}$ \\
\hline Air mass flow & $q_{\max }$ & Maximum air mass flow & $q^{*}=\frac{q}{q_{\max }}$ \\
\hline Volume flow & $Q_{\max }=\frac{V_{h}}{T_{\min }}=A_{h} \bar{u}$ & Maximum oil volume flow & $Q^{*}=\frac{Q}{Q_{\max }}$ \\
\hline Air mass & $m_{p \max }=\operatorname{roupn} * V p$ & Maximum air mass & $m^{*}=\frac{m}{m_{p \max }}$ \\
\hline
\end{tabular}




\begin{tabular}{|c|c|c|c|}
\hline Displacement & $L$ & Piston stroke & $x^{*}=\frac{x}{L}$ \\
\hline Volume & $V_{p \max }=L \cdot A_{p}$ & Maximum volume of pneumatic chamber & $V^{*}=\frac{V}{V_{p \max }}$ \\
\hline $\begin{array}{c}\text { Power of } \\
\text { compressed } \\
\text { air }\end{array}$ & $P_{\mathrm{p} \max }=\frac{p_{a} q_{\max }}{\rho_{a}} \ln \frac{p_{s}}{p_{a}}$ & Maximum power of compressed air & $P_{p}^{*}=\frac{P_{p}}{P_{\mathrm{pmax}}}$ \\
\hline $\begin{array}{c}\text { Power of } \\
\text { hydraulic oil }\end{array}$ & $P_{h \max }=p_{s} Q_{\max }$ & $\begin{array}{c}\text { Maximum power of hydraulic oil when } \\
\text { its pressure equals the supply pressure } \\
\left(p_{s}\right)\end{array}$ & $P_{h}^{*}=\frac{P_{h}}{P_{\mathrm{h} \max }}$ \\
\hline
\end{tabular}

Dimensionless continuity equations of pneumatic syste

$$
q^{*}= \begin{cases}\frac{p_{p u}^{*}}{\sqrt{\theta_{u}^{*}}}\left[\left(\frac{p_{d}^{*}}{p_{u}^{*}}\right)^{\frac{2}{\kappa}}-\left(\frac{p_{d}^{*}}{p_{u}^{*}}\right)^{\frac{\kappa+1}{\kappa}}\right] & \frac{p_{d}^{*}}{p_{u}^{*}}>0.528 \\ \frac{p_{p u}^{*}}{\sqrt{\theta_{u}^{*}}} & \frac{p_{d}^{*}}{p_{u}^{*}} \leq 0.528\end{cases}
$$

\section{Dimensionless energy equations of pneumatic system}

The dimensionless energy equations for the discharge side and the charge side can be written as follows:

$$
\begin{aligned}
m^{*} \frac{d \theta^{*}}{d t^{*}} & =\frac{s^{*}}{S_{p}^{*} T_{d}^{*}}\left(1-\theta^{*}\right)+(k-1)\left(p^{*} u^{*}-q^{*} \theta^{*}\right) \\
m^{*} \frac{d \theta^{*}}{d t^{*}} & =\frac{s^{*}}{S_{p}^{*} T_{c}^{*}}\left(1-\theta^{*}\right)+(k-1)\left(q^{*} \theta^{*}-p^{*} u^{*}\right)
\end{aligned}
$$

In the equations, $T_{d}^{*}=\frac{T_{d}}{T_{m t n}} ; T_{d}=\frac{C_{v} m_{p m a x}}{S_{p} h_{d}} ; S_{p}=2 A_{p}+2 L \sqrt{\pi A_{p}}$.

The dimensionless maximum heat transfer area can be given as:

$$
S_{p}^{*}=\frac{2 A_{p}+2 L \sqrt{\pi A_{p}}}{A_{p}}=2+2 L \sqrt{\frac{\pi}{A_{p}}}
$$

For the charge side:

$$
\begin{gathered}
T_{c}^{*}=\frac{T_{c}}{T_{\min }} \\
T_{c}=\frac{C_{v} m_{p \max }}{S_{p} h_{c}},
\end{gathered}
$$

Dimensionless state equation of pneumatic system

$$
\frac{d p_{p}^{*}}{d t^{*}}=\frac{p_{p}^{*}}{\theta_{p}^{*}} \frac{d \theta_{p}^{*}}{d t^{*}}+\frac{\theta_{p}^{*} q^{*}}{V_{p}^{*}}-\frac{p_{p}^{*} u^{*}}{V_{p}^{*}}
$$

\section{Motion equations}

$$
\frac{d^{2} x^{*}}{d\left(t^{*}\right)^{2}}= \begin{cases}\left(\frac{1}{T_{f}^{*}}\right)^{2}\left(p_{l h}^{*} \cdot A_{h}^{*}-p_{r h}^{*} \cdot A_{h}^{*}+p_{l p}^{*}-p_{r p}^{*}-F_{f}^{*}\right) & x^{*} \neq 0,1 \\ 0 & x^{*}=0,1\end{cases}
$$


In the equations,

$$
F_{f}= \begin{cases}F_{s}^{*} & u^{*}=0 \\ F_{c}^{*}+C^{*} u^{*} & u^{*} \neq 0\end{cases}
$$

$F_{s}^{*}$ : dimensionless maximum static friction force;

$F_{c} *$ : dimensionless Coulomb friction force;

$C^{*}$ : dimensional viscous friction force coefficient.

And

$$
\begin{aligned}
& F_{s}^{*}=\frac{F_{s}}{P_{s} A_{p}} \\
& F_{s}^{*}=\frac{F_{c}}{P_{s} A_{p}} \\
& C^{*}=\frac{C u_{0}}{P_{s} A_{p}} \\
& \mathrm{~J}=\left(\frac{1}{T_{f}^{*}}\right)^{2} \\
& \mathrm{~J}=\frac{T_{p}^{2} P_{s} A_{p}}{L M} \\
& T_{f}=\sqrt{\frac{M L}{A_{p} P_{s}}} \\
& T_{f}^{*}=\frac{T_{f}}{T_{\min }}
\end{aligned}
$$

Dimensionless pressure equations of hydraulic system

In the equations,

$$
\begin{gathered}
\frac{d p_{\text {Ih }}^{*}}{d t^{*}}=\beta^{*}\left(A h^{*} \cdot Q_{\text {Ihin }}^{*}-A h^{*} \cdot Q_{\text {Ihout }}^{*}-u^{*}\right) \\
\frac{d p_{r h}^{*}}{d t^{*}}=\beta^{*}\left(Q_{\text {rhin }}^{*}-Q_{\text {rhout }}^{*}+u^{*}\right)
\end{gathered}
$$

$$
\beta^{*}=\frac{\beta}{p_{s}}
$$

Dimensionless flow equations of hydraulic system

$$
\begin{gathered}
Q_{\text {hin }}{ }^{*}=A_{\text {ehin }}^{*} \sqrt{\frac{p_{u h}^{*}-p_{d h}^{*}}{p_{f}^{*}-p_{a}^{*}}} \\
Q_{\text {hout }}{ }^{*}=A_{\text {ehout }}^{*} \sqrt{\frac{p_{u h}^{*}-p_{d h}^{*}}{p_{f}^{*}-p_{a}^{*}}} \\
A_{\text {ehin }}^{*}=\frac{A_{\text {ehin }}}{A_{\text {ep }}} \\
A_{\text {ehout }}^{*}=\frac{A_{\text {ehout }}}{A_{e p}}
\end{gathered}
$$

Power of pneumatic system

$$
P_{p}^{*}=\frac{q_{p}^{*}\left[\ln \frac{p_{p}^{*}}{p_{a}^{*}}+\frac{k}{k-1}\left(\theta_{p}^{*}-1-\ln \theta_{p}^{*}\right)\right]}{\ln \frac{1}{p_{a}^{*}}}
$$

Power of hydraulic system 


$$
P_{h}^{*}=p_{h}^{*} Q_{h}^{*}
$$

\section{Dimensionless analysis of key parameter of the air-powered vehicle}

We set key parameters influencing the efficiency is dimensional output oil's pressure, including the stroke of the piston and the dimensional area ratio of the piston on the pneumatic chambers and hydraulic chambers. To explore the relationship between the each parameter and the efficiency, we take the control variable method that changes one variable while others keep constant.

\section{Influence of dimensionless output oil's pressure}

The dimensionless out put power and efficiency is studied when the stroke of the piston and dimensional area ratio is set at 0.10 and 2, and the dimensionless 0 u t put oil's pressure is set at 2, $2.29,2.57,2.86$ and 3.14. Figure 6 describes the dimensional output power under the difference situation and the figure 7 shows the value of efficiency when dimensionless 0 u t put oil's pressure varies.

As we can see from the figure 2 and 3, the dimensional output power decreases with the increase of the dimensionless 0 u t put oil's pressure, but it is also obviously that efficiency increases in this process. This is because more expansion energy is used in this procession. Considering the dimensionless 0 ut put power and efficiency, we suggested that dimensionless 0 u t put oil's pressure is range from $20-22$.

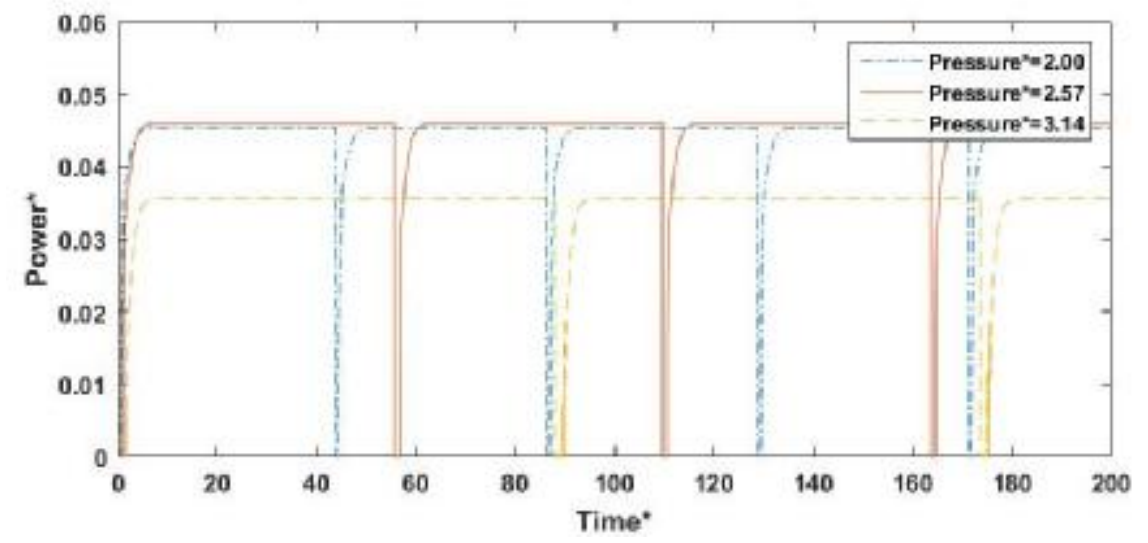

Fig.2. Dimensionless power*-time* curves.

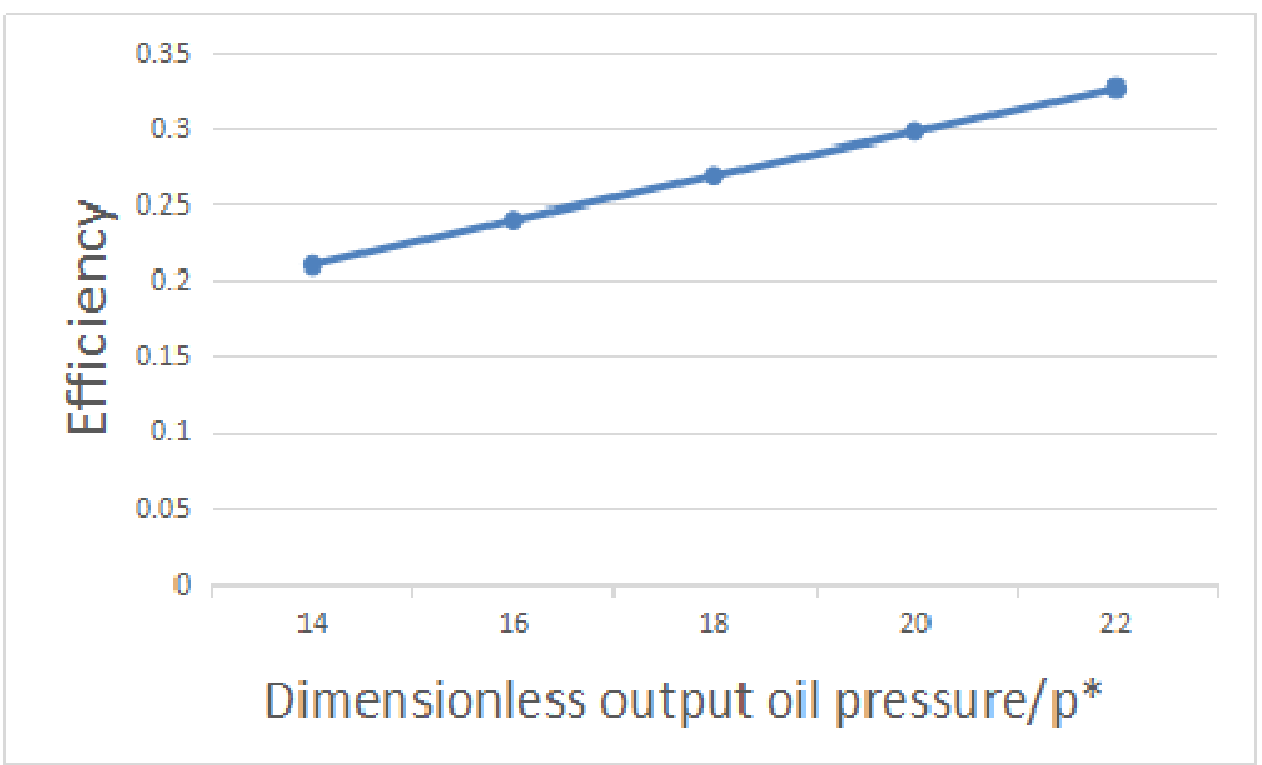

Fig.3. Efficiency curves. 


\section{Influence of piston's stroke}

Piston's stoke is up to chamber's size. The dimensionless output power and efficiency is studied at the time dimensional output pressure and dimensional area ratio is set at 2.57 and 2, and the stroke of the piston is set at $0.08 \mathrm{~m}, 0.09 \mathrm{~m}, 0.10 \mathrm{~m}, 0.11 \mathrm{~m}$ and $0.12 \mathrm{~m}$. Figure 8 describes the dimensional output power under the difference situation. Figure 9 shows relationship of efficiency and stroke of pistons.

As can be seen in figure 4 and 5, dimensional output power nearly keeps constant with the increase of the piston's stroke, and this situation is suit to the efficiency. This is because the speed of doing work is not varied nearly when the stroke of the piston increase. So the stroke of the piston is mainly up to the size of machine, and we can select the value of the stroke according to the actual needs. And during the process of our study, the value of the stroke floating around $0.10 \mathrm{~m}$ is a better choice.

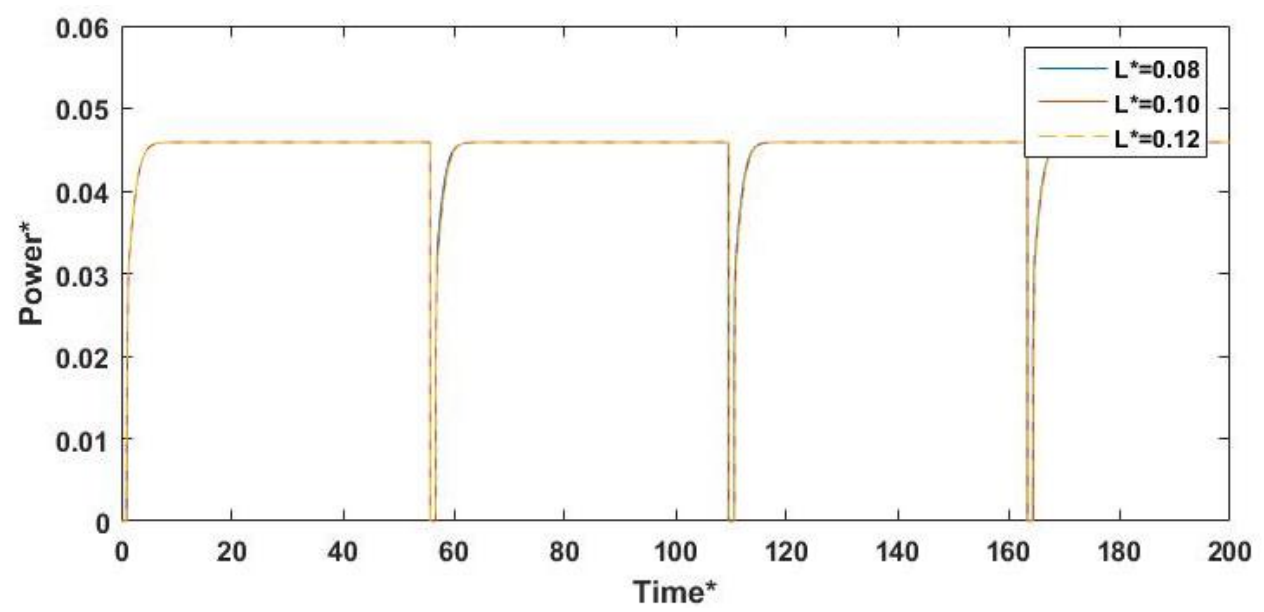

Fig.4. Power-time curves

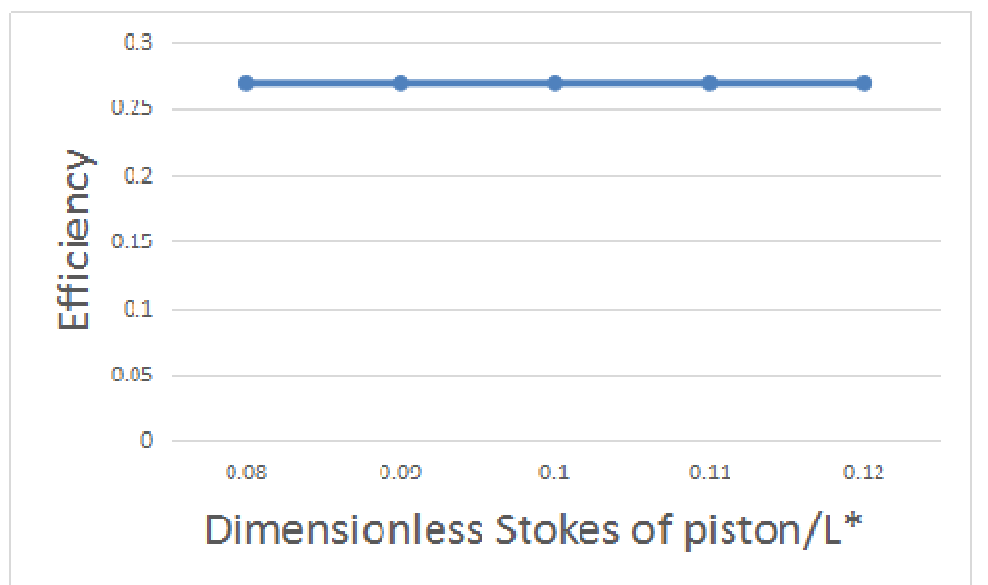

Fig.5. Efficiency curves

\section{Influence of dimensional piston area ratio}

The dimensional area ratio of the piston is that the effective area of the piston in pneumatic chamber divided by the effective area of the piston in pneumatic chamber. The dimensional output power and efficiency is studied when the stroke of the piston and dimensional output pressure is set at $0.10 \mathrm{~m}$ and 2.57 , and then dimensional piston area ratio is set 2.86, 2.50, 2.22, 2 and 1.82. Figure 10 describes the dimensional output power under the difference situation. Figure 11 shows the value of efficiency when the dimensional area ratio of the piston varies.

From the picture 6 and 7, we would know that enlarging the dimensional area ratio will lead to output power increasing, but the efficiency decreases in this process. Because the driving chamber's 
area is bigger, the more expansion energy will be wasted when the pneumatic chamber is connected with the atmosphere. In order to maximize efficiency and power at the same time, the dimensional area ratio of the piston can be range from 2.50 to 2.86 , which assure that efficiency would exceed $27.5 \%$.

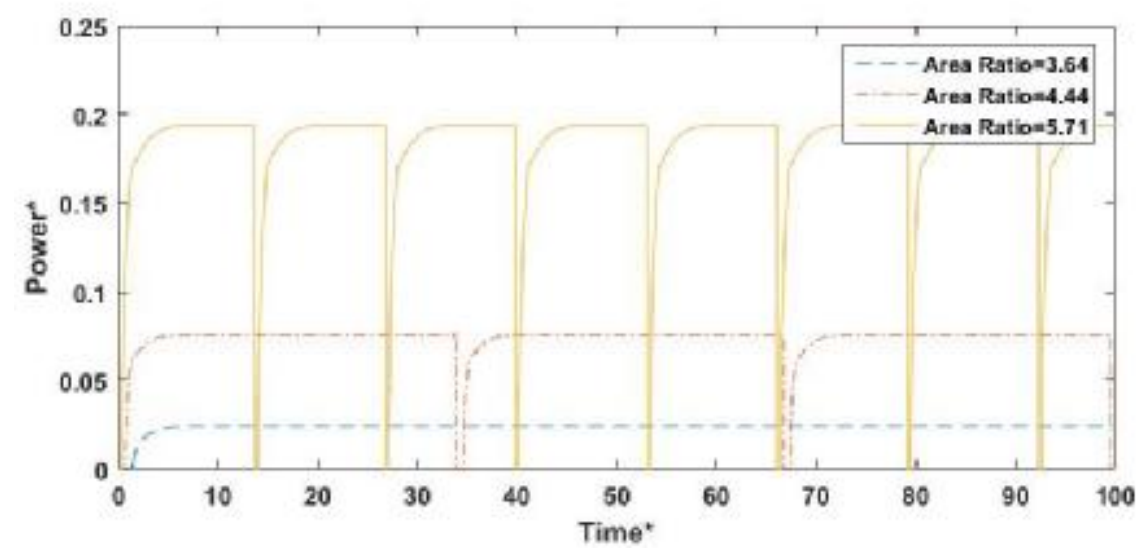

Fig.6. Power-time curves

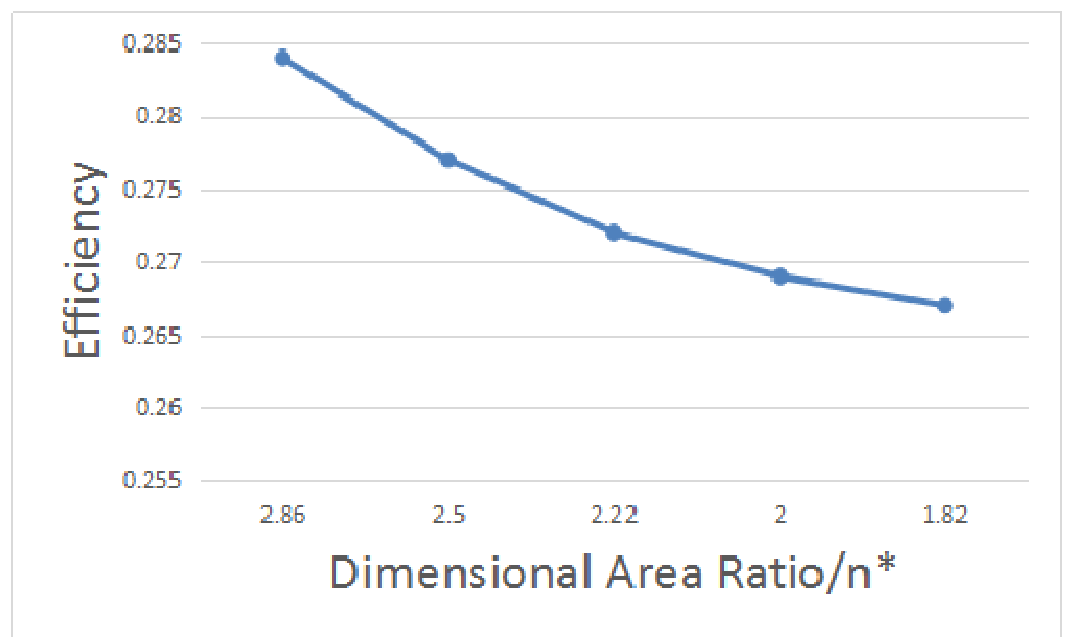

Fig.7. Efficiency curves

\section{Conclusions}

We build up a mathematical model of the hydro-pneumatic transformer. To optimize the efficiency performance, we study the key parameters influencing the dimensional output power and efficiency. And some conclusions can be draw as follows:

(1).Dimensional output power and efficiency increases with the increase of the dimensional output oil's pressure. Considering the output power and efficiency, we suggested that input pressure of compressed air is range from 20-22.

(2). The dimensional output power and efficiency keeps constant nearly with the increase of the piston's stroke. So the stroke of the piston is mainly up to acturally need.

(3). Enlarging the dimensional area ratio will lead to dimensional output power increasing, but the efficiency decreases in this process. And the dimensional area ratio of the piston can be range from 2.50 to 2.86 in order to maximize efficiency and power at the same time, which assure that efficiency would exceed $27.5 \%$.

This paper can be a reference in designing and optimizing of the HP transformer used on air-powered vehicle.

\section{Acknowledgements}

Supported by National Natural Science Foundation of China (Grant No. 51375028). 


\section{References}

[1] Shi, Y., Wang, Y., Liang, H., \& Cai, M. (2016). Power characteristics of a new kind of air powered vehicle.International Journal of Energy Research, 40(8), pp: 1112-1121.

[2] Cai, M., Wang, Y., Shi, Y., \& Liang, H. (2016). Output dynamic control of a late model sustainable energy automobile system with nonlinearity. Advances in Mechanical Engineering, 8(11), 1687814016672784.

[3] Shen, D., Zhang, Q., \& Shi, Y. (2016). Dynamic Characteristics of Mechanical Ventilation System of Double Lungs with Bi-Level Positive Airway Pressure Model. Computational and mathematical methods in medicine, 2016.

[4] Shi, Y., Niu, J., Cai, M., \& Xu, W. (2015). Dimensionless study on dynamics of pressure controlled mechanical ventilation system. Journal of Mechanical Science and Technology, 29(2), 431.

[5] O. Takeuchi, et al., Characteristics Analysis of Expanding-type Booster. Proceedings of 1995 Autumn Symposium on Hydraulics and Pneumatics, Tokyo (Japan):1995 (in Japanese). 